\title{
Le traitement orthodontique peut-il générer des algies et/ou dysfonctionnements articulaires ou musculaires (ADAM) ? Une revue de littérature
}

\author{
Sarah Gebelle-Chauty ${ }^{1 *}$, Olivier Robin ${ }^{2}$, Yassine Messaoudi ${ }^{1}$, Jean-Jacques Aknin ${ }^{1}$ \\ 1 Département d'Orthopédie Dento-Faciale, Faculté d'Odontologie, 11 rue Guillaume Paradin, 69372 Lyon Cedex 08, France \\ 2 Consultation ADAM, Service d'Odontologie, CHU de Lyon, 6-8 place Depéret, 69365 Lyon Cedex 07, France
}

MOTS CLÉS :

ATM /

Dysfonction

temporo-mandibulaire /

Orthodontie

\section{KEYWORDS:}

Temporo-mandibular joint /

Temporo-mandibular

disorders /

Orthodontics

\begin{abstract}
RÉSUMÉ - Longtemps considérée comme fortement responsable des algies et/ou dysfonctionnements articulaires ou musculaires (ADAM), l'occlusion est aujourd'hui considérée comme un des facteurs participant à l'étiologie, parmi d'autres. Les études récentes, revues de littérature ou méta-analyses, études longitudinales avec suivi d'enfants traités sur plusieurs années, concluent à l'absence de risque pour un traitement orthodontique de déclencher des ADAM. L'apparition de signes d'ADAM au cours d'un traitement orthodontique doit être replacée dans le contexte épidémiologique caractérisé par une forte augmentation de leur prévalence au cours de la période de l'adolescence. En conclusion, si les traitements orthodontiques ne peuvent être considérés comme des facteurs étiopathogéniques avérés des ADAM, il n'existe pas, à l'inverse, d'arguments scientifiques permettant de justifier l'indication de traitements orthodontiques dans le seul but de traiter les ADAM.
\end{abstract}

ABSTRACT - Do orthodontic treatment generate temporomandibular disorders? A review. While considered for years to play the primary role in the etiology of temporo-mandibular joint disturbances (TMD), occlusal discrepancies are now considered to be just one causative factor among many. Recent studies, literature reviews or meta-analyses, and longitudinal studies with follow-up of children treated for many years all conclude that there is no risk of orthodontic treatment giving rise to episodes of temporo-mandibular disorders. The signs of TMD appearing during the course of orthodontic treatment should be considered in the context of the epidemiology of the disorder, which is characterized by a strong increase in its occurrence during adolescence. In conclusion, it should be stated that if orthodontic treatment can no longer be considered as one of the etiopathogenic factors in the TMD complex, there are no scientific arguments to justify the converse, that there are indications for orthodontic treatment whose sole goal would be the treatment of TMD.

\section{Introduction}

L'existence préalable ou la survenue de symptômes d'algies et/ou de dysfonctionnements articulaires ou musculaires (ADAM) au cours d'un traitement orthodontique (craquements, douleur, blocages articulaires, limitation fonctionnelle, gêne à la mastication) sont des sujets de préoccupation importants pour les orthodontistes. Historiquement, le débat concernant les relations entre ADAM et traitements orthodontiques a été lancé en 1987 aux États-Unis, à la suite d'un procès intenté par un patient contre son orthodontiste. Depuis ce procès, qui a conclu à la responsabilité de l'orthodontiste, un grand nombre d'études a été réalisé afin de clarifier cette relation, notamment le risque éventuel pour un traitement orthodontique de déclencher des ADAM [53].

* Auteur pour correspondance : schauty@aliceadsl.fr 
En réalité, les résultats de ces études doivent être replacés dans le contexte épidémiologique de l'apparition des ADAM en fonction de l'âge. Même si les données épidémiologiques varient selon les auteurs [7], notamment en raison de la disparité des critères de diagnostic [82], elles montrent que la prévalence des signes d'ADAM peut être élevée chez les enfants et les adolescents (jusqu'à 50 \% selon Köhler, et al. [47]). Mais surtout, elles révèlent qu'indépendamment de tout traitement orthodontique, cette prévalence augmente fortement au cours de la période $10-19$ ans $[9,15,42,54,63,87]$.

Ainsi, Macfarlane, et al. [54] ont montré, sur une population de 1018 enfants suivis sur plusieurs années, que le pourcentage d'enfants présentant des ADAM est multiplié par 5 entre 11 ans $(3,2 \%)$ et 19 ans $(17,6 \%)$.

De même, la prévalence des déplacements discaux antérieurs, à l'origine des craquements et blocages articulaires, qui se situe autour de $4 \%$ à l'âge de 4 ans, augmente de façon importante jusqu'à 15 ans pour atteindre $20 \%$ chez les garçons et $35 \%$ chez les filles [42].

Ainsi, l'augmentation spontanée de la prévalence des signes d'ADAM et leur fluctuation au cours de la tranche d'âge où est effectuée la grande majorité des traitements orthodontiques (10-19 ans), rend d'emblée difficile et aléatoire toute tentative de corrélation entre ADAM et orthodontie [38].

\section{2. Étiopathogénie des ADAM [74]}

Les algies et dysfonctionnements de l'appareil manducateur regroupent un ensemble de symptômes qui concernent principalement les articulations temporo-mandibulaires (ATM) et les muscles masticateurs avec, dans certains cas, la présence de signes associés tels que les céphalées, migraines, ou acouphènes.

Les ADAM peuvent donc être regroupés en deux grandes familles : articulaire et musculaire.

Les ADAM articulaires se traduisent par des douleurs inflammatoires (capsulite, synovite, rétrodiscite), des troubles fonctionnels (limitation d'ouverture, déviation à l'ouverture...) et/ou des bruits articulaires (claquements ou craquements, crépitements). Ces symptômes résultent de traumatismes articulaires, de dysfonctionnements du complexe condylo-discal (luxations discales réductibles ou non réductibles, adhérence discale) ou d'atteintes dégénératives des surfaces articulaires (arthrite, arthrose).

Les ADAM musculaires se traduisent par des douleurs (myalgies), des sensations de fatigue ou de tension localisées au niveau des muscles masticateurs et parfois étendues à un territoire cervico-céphalique.

Historiquement, c'est Costen [16], médecin ORL, qui a suggéré (à partir de l'observation de 13 patients) que c'est la perte des molaires qui était à l'origine des douleurs musculo-articulaires, mais aussi des signes cliniques parfois associés comme les céphalées, les acouphènes, les vertiges et les problèmes sinusiens.

Cependant, la corrélation entre la perte du calage postérieur et la compression de la région auriculaire et des ATM par un enfoncement du condyle dans la fosse mandibulaire n'a pu être démontrée.

Depuis, plusieurs facteurs ont été incriminés pour tenter d'expliquer l'étiologie des ADAM qui est probablement multifactorielle: facteurs occlusaux, posturaux, psychosociaux, traumatiques et systémiques [35].

\subsection{Facteurs occlusaux}

Pendant longtemps (des années 1950 à 1980), on a attribué aux troubles occlusaux un rôle essentiel dans le déclenchement et l'entretien des ADAM. Ce concept reposait sur le soulagement de certaines douleurs musculo-articulaires par la suppression de contacts occlusaux jugés nocifs (prématurités, interférences en propulsion, en latéralité).

Cette conception est aujourd'hui largement remise en cause en l'absence de preuves scientifiques démontrant une relation évidente entre troubles de l'occlusion et ADAM [14,28,31,36,46,53,55,56,83].

Pullinger, et al. [70] ont montré que cinq conditions occlusales (infraclusion antérieure, recouvrement ou surplomb incisif $>7 \mathrm{~mm}$, articulé croisé molaire unilatéral, décalage entre relation centrée et position d'intercuspidation maximale $>3 \mathrm{~mm}$ et édentement postérieur) seraient plus fréquemment rencontrées chez les patients présentant des ADAM que chez des sujets contrôles. Certaines de ces associations n'ont cependant pas été confirmées par d'autres études.

Parmi ces facteurs, l'articulé croisé molaire unilatéral, condition occlusale retrouvée chez $12 \%$ des enfants de 10 à 16 ans [27], a fait l'objet d'une attention plus particulière chez l'enfant, car sa correction 
orthodontique pourrait se justifier dans le cadre de la prévention des ADAM. Cependant, deux études récentes n'ont pas permis de confirmer l'existence, chez l'enfant, d'une association significative entre un articulé croisé molaire unilatéral et la présence de craquements articulaires [27] et de déplacement discal à l'IRM [67].

\subsection{Facteurs posturaux}

Il a été suggéré que certains désordres musculoarticulaires pourraient être la conséquence d'un trouble de la posture corporelle (asymétrie, inclinaison du bassin, différence de longueur des jambes, posture céphalique antérieure...). Mais, à ce jour, l'étiologie posturale des ADAM est toujours dans l'attente d'une validation scientifique, la relation entre ADAM et posture cervico-céphalique n'étant pas clairement établie [4]. Les études menées dans ce domaine souffrent souvent d'insuffisances méthodologiques et aboutissent à des résultats contradictoires $[58,62]$.

\subsection{Facteurs psycho-sociaux}

Limportance des facteurs psychologiques (stress, anxiété, dépression) dans l'évolution et l'entretien des ADAM est largement reconnue [29,34]. Les patients ADAM présentent souvent un profil stressé ou anxieux [65], de telle sorte que leurs symptômes pourraient être la conséquence d'une projection de leur trouble psychique sur les structures musculoarticulaires (somatisation) [11, 20, 29, 85, 93].

\subsection{Facteurs traumatiques}

Plus fréquemment associés aux dysfonctionnements articulaires, les traumatismes que peut subir l'appareil manducateur sont regroupés en macrotraumatismes et microtraumatismes, ces derniers étant assimilables aux parafonctions.

Les macrotraumatismes regroupent les traumatismes directs de la face [94], les traumatismes indirects comme l'entorse cervicale $[45,86]$ et toute ouverture buccale forcée et prolongée survenant, par exemple, lors d'un bâillement, d'une anesthésie générale (intubation), de l'extraction des dents de sagesse, de séances de soins dentaires, du fait d'un possible étirement des attaches ligamentaires ou discales de l'ATM.
Les activités parafonctionnelles à type de serrement des dents $[13,33,81]$, bruxisme $[6,75]$, mastication fréquente de chewing-gum, tics de morsure des ongles, des stylos, des lèvres, de la langue [30], mastication unilatérale [73] sont autant de microtraumatismes répétés pour l'appareil manducateur, qui n'a pas été conçu pour supporter des contraintes prolongées en dehors des repas.

\subsection{Facteurs systémiques}

Les dysfonctionnements articulaires peuvent être favorisés par des conditions prédisposantes telles que l'hyperlaxité ligamentaire [21], plus fréquente chez la femme, et certaines maladies génétiques $\mathrm{du}$ tissu conjonctif (syndrome de Marfan ou d'Ehlers-Danlos) responsables d'une hyperlaxité articulaire [18].

De même, les pathologies articulaires systémiques telles que l'arthrite rhumatoïde [37] ou certaines maladies auto-immunes (lupus érythémateux, sclérodermie...) [44] peuvent avoir des localisations au niveau des ATM.

\section{Traitements orthodontiques et ADAM}

Depuis les années 1980, de nombreuses études sont régulièrement publiées sur les relations qui peuvent exister entre traitements orthodontiques et ADAM avec l'objectif de pouvoir répondre aux deux questions fondamentales suivantes:

1. Les traitements orthodontiques peuvent-ils favoriser l'apparition des ADAM?

2. Les traitements orthodontiques sont-ils indiqués pour traiter les ADAM?

Les premières études $[17,50,78,79]$ avaient déjà montré qu'il n'y avait pas de différence concernant la prévalence des ADAM entre des sujets ayant subi un traitement orthodontique et des sujets n'ayant pas eu de traitement orthodontique.

Ces conclusions ont été confirmées par différentes études longitudinales, notamment celles de Rendell, et al. [71], Kremenak, et al. [48] et Hirata, et al. [39] à partir du suivi de patients ayant bénéficié d'un traitement orthodontique. Ainsi, un traitement réalisé pendant l'adolescence ne modifierait pas le risque d'apparition ultérieure d'ADAM. 


\subsection{Influence du type d'appareil}

\subsubsection{Disjonction maxillaire}

Il est admis qu'un environnement buccal équilibré dans le cadre de relations dento-squelettiques harmonieuses et fonctionnelles contribue à l'entretien de la santé des ATM et minimise le risque d'apparition de dysfonctions. Cet environnement peut être amélioré par une expansion du maxillaire associée à un traitement orthodontique. Selon Arat, et al. [3], la disjonction maxillaire rapide ne semble être ni un facteur de risque, ni un moyen de prévention des dysfonctionnements articulaires. Lanalyse de clichés issus d'imagerie par résonance magnétique (IRM), effectués après disjonction palatine rapide (Type Hyrax), montre que la position des condyles reste inchangée.

\subsubsection{Activateurs de croissance}

D’après Popowich, et al. [68], concernant les effets de l'utilisation de bielles de Herbst sur la morphologie des ATM, il apparaît que les changements de la position du condyle dans la fosse temporale sont minimes et ne sont pas cliniquement significatifs. Ainsi, la nature du remodelage du condyle et de la fosse temporale n'a pu être caractérisée de même que le changement de position du disque n'a pu être établi [69].

Le traitement par bielle de Herbst engendre des perturbations mineures et temporaires du système masticatoire. Celles-ci apparaissent surtout au début du traitement, les patients rapportant des difficultés à la mastication durant les 7 à 10 premiers jours [68]. Ruf, et al. [76] avaient également montré que la prévalence des dysfonctions articulaires n'était pas plus élevée chez les patients ayant reçu un traitement d'une malocclusion de classe II par bielle de Herbst que chez des patients non traités.

Finalement, les études réalisées à court et à long terme sur les effets des traitements par bielles de Herbst ont conclu à l'absence d'effet délétère sur le fonctionnement des ATM $[68,76,77]$. Une amélioration des symptômes a même été constatée chez les patients souffrant de problèmes articulaires avant traitement, ce qui s'expliquerait par un remodelage du condyle et de la fosse temporale [76].

\subsubsection{Force extra-buccale}

\section{- Classe III}

D'après Deguchi, et al. [19], l'utilisation d'une fronde mentonnière n'augmente pas la prévalence des dysfonctionnements articulaires, ni pendant, ni après traitement.

Des conclusions similaires sont rapportées par Rey, et al. [72] pour les patients présentant une classe III squelettique, traités par force extra-buccale à appui cervical et traitement multi-attaches, par rapport aux patients en classe I squelettique, traités par multi-attaches ou encore par rapport aux patients non traités.

\section{- Classe II}

Une amélioration fonctionnelle est même constatée chez les patients qui présentaient initialement une classe II, division 1 .

Finalement, ce sont les patients chez qui préexistaient des signes d'ADAM qui s'avèrent les plus susceptibles de développer des ADAM après le traitement [43].

\subsubsection{Multi-attache}

Dibbets, et al. ont comparé les traitements fonctionnels et fixes [23] et ont suivi 171 patients traités soit par thérapeutique fixe, soit par thérapeutique fonctionnelle: les sujets traités par thérapeutique fixe avaient un plus grand pourcentage de symptômes de dysfonction temporo-mandibulaire que ceux traités par thérapeutique fonctionnelle. Mais cette différence disparaît au bout de 20 ans.

- Influence des extractions

Witzig, et al. [90] ou encore Wyatt [92] ont suggéré que l'avulsion des prémolaires entraînait une perte de dimension verticale et une version palatine des incisives maxillaires qui favoriseraient un recul de la mandibule et une position plus postérieure des condyles, à l'origine de dysfonctionnements articulaires.

Ces observations n'ont cependant pas été confirmées par plusieurs études ultérieures [59] par une comparaison de la prévalence des signes d'ADAM (notamment des bruits articulaires) avant et après traitements réalisés avec ou sans extraction de prémolaires. Ainsi, les auteurs concluent que les traitements orthodontiques réalisés avec extractions dentaires ne constituent pas un facteur de risque plus 
élevé d'apparition d'ADAM, ni d'aggravation de la sévérité des symptômes et des signes existants avant le traitement $[5,8,22,23,32,38,48,51,52,57,66,80]$.

- Influence des élastiques

Il ne semble pas y avoir de corrélation entre l'utilisation d'élastiques, quelle que soit leur indication (classe II ou III, inter- ou intra-arcade) et le risque de développement d'ADAM [64].

\subsection{Traitements chirurgico-orthodontiques}

Les conséquences éventuelles de la chirurgie orthognatique sur les signes et symptômes d'ADAM a été récemment évaluée par Abrahamsson, et al. [1] dans une revue de littérature. Cependant, sur les 467 articles publiés sur le sujet entre 1966 et 2006, seuls trois d'entre eux répondaient aux critères d'inclusion définis par les auteurs qui n'ont pu conclure qu'à la nécessité d'entreprendre d'autres études plus fiables scientifiquement. Des conclusions similaires ont été faites par Cascone, et al. [12] à partir d'une revue de littérature effectuée sur les 20 années précédentes. Ces auteurs soulignent la difficulté rencontrée pour comparer les études entre elles du fait d'une trop grande disparité des méthodologies utilisées et des traitements effectués. Ils concluent néanmoins à la nécessité d'entreprendre un traitement spécifique en présence d'ADAM, ce qui signifie que la chirurgie orthognathique ne semble pas indiquée dans le seul but de traiter les ADAM. Cette opinion est également partagée par Al-Riyami, et al. [2] en conclusion d'une autre revue de littérature sur ce sujet, même si les auteurs rapportent que, dans certains cas, le traitement chirurgico-orthodontique peut apporter une certaine amélioration des symptômes d'ADAM.

Il ressort finalement de ces récentes revues de littérature qu'il est impossible de conclure sur les effets des traitements chirurgico-orthodontiques sur les ADAM, du fait d'une trop grande hétérogénéité des études et de l'insuffisance de leur rigueur méthodologique.

Les points de vue des auteurs sur ce sujet sont effectivement divergents. Certains auteurs, tels que Wolford, et al. [91] et Borstlap, et al. [10] estiment que les traitements chirurgicoorthodontiques peuvent conduire à une aggravation des dysfonctions articulaires, en favorisant des lésions au niveau de l'ATM.

Borstlap, et al. [10] constatent ainsi des limitations dans les mouvements d'ouverture et de latéralité après l'intervention qui pourraient être la conséquence de l'inflammation post-opératoire. Une amélioration est observée dans les 24 mois postopératoires [95], mais il persiste une diminution de l'amplitude de l'ouverture buccale de 1 à $2 \mathrm{~mm}$ en moyenne à 2 ans [10] .

À l'inverse, des études comme celles de White et Dolwick [88] rapportent des améliorations en postopératoire. Sur les 37 patients qui présentaient des symptômes de dysfonction avant la chirurgie, 33 d'entre eux ont constaté une amélioration. Pour les quatre autres patients, les symptômes étaient inchangés ou s'étaient aggravés.

Certaines études rapportent un soulagement des douleurs articulaires, musculaires et des céphalées [2]. Les résultats sur les bruits articulaires (craquements) sont controversés.

La plupart des études qui ont rapporté une amélioration après la chirurgie concernent des patients qui présentaient une classe II squelettique. En revanche, les effets sont plus fluctuants et plus imprévisibles dans les cas de classe III [26].

Al-Riyami, et al. [2] s'interrogent sur l'origine des améliorations qui peuvent être observées : sont-elles le résultat de la chirurgie elle-même ou sont-elles dues à un effet placebo du fait du changement de la perception de leur image par les patients? Turner, et al. [84] ont constaté que l'effet placebo influence le vécu du patient, quelle que soit la nature du traitement effectué, lorsque le patient et le praticien sont convaincus de son efficacité.

Finalement, en l'absence d'un nombre suffisant d'études scientifiquement fiables, il est difficile de conclure sur les relations entre traitements chirurgico-orthodontiques et ADAM. Il ressort cependant qu'une chirurgie orthognatique ne devrait pas être envisagée dans le seul but d'améliorer les dysfonctions articulaires. Le patient doit être informé sur l'impossibilité de prédire la nature des effets d'une telle chirurgie sur les symptômes d'ADAM qui peuvent évoluer dans le sens d'une amélioration mais aussi d'une aggravation.

\section{Conclusion}

Les études récentes, revues de littérature ou métaanalyses [41,60], études longitudinales avec suivi d'enfants traités sur plusieurs années [24, 40, 54, 61], concluent à l'absence de risque pour un traitement orthodontique de déclencher des ADAM. 
Lapparition de signes d'ADAM au cours d'un traitement orthodontique doit être replacée dans le contexte épidémiologique caractérisé par une forte augmentation de leur prévalence au cours de la période 10-20 ans. Une attention particulière devra néanmoins être portée chez un enfant qui présenterait des signes d'ADAM avant un traitement ou des facteurs favorisants comme une hyperlaxité ligamentaire ou des activités parafonctionnelles. Dans ce cas, il sera impératif de supprimer les parafonctions et de minimiser l'intensité des forces qui pourraient être transmises aux ATM, notamment par le biais d'élastiques intermaxillaires ou de forces extra-orales.

En conclusion, si les traitements orthodontiques ne peuvent être considérés comme des facteurs étiopathogéniques avérés des ADAM, il n'existe pas, à l'inverse, d'arguments scientifiques permettant de justifier l'indication de traitements orthodontiques dans le seul but de traiter les ADAM.

\section{Bibliographie}

[1] Abrahamsson C, Ekberg EC, Henrikson T, Bondemark L. Alterations of temporomandibular disorders before and after orthognathic surgery. Angle Orthod 2007;77:729-734.

[2] Al-Riyami S, Cunnigham SJ, Moles DR. Orthognathic treatment and temporomandibular disorders: a systematic review. Part 2. Signs and symptoms and meta-analyses. Am J Orthod Dentofacial Orthop 2009;136:626. el-e16.

[3] Arat FE, Arat ZM, Tompson B, Tanju S. Muscular and condylar response to rapid maxillary expansion. Part 3: Magnetic resonance assessment of condyle-disc relationship. Am J Orthod Dentofacial Orthop 2008;133:830836.

[4] Armijo Olivo SA, Bravo J, Magee DJ, Thie NMR, Major PW, Flores-Mir C. The association between head and cervical posture and temporomandibular disorders: a systematic review. J Orofac Pain 2006;20:9-23.

[5] Artun J, Hollender LG, Truelove EL. Relationship between orthodontic treatment, condylar position, and internal derangement in the temporomandibular joint. Am J Orthod Dentofacial Orthop 1992;101:48-53.

[6] Baba K, Haketa T, Sasaki Y, Ohyama T, Clark GT. Association between masseter muscle activity levels recorded during sleep and signs and symptoms of temporomandibular disorders in healthy young adults. J Orofac Pain 2005;19:226-231.

[7] Barbosa Tde S, Miyakoda LS, Pocztaruk Rde L, Rocha CP, Gavião MB. Temporomandibular disorders and bruxism in childhood and adolescence: review of the literature. Int J Pediatr Otorhinolaryngol 2008;72:299-314.

[8] Beattie JR, Paquette DE, Johnston LE Jr. The functional impact of extraction and nonextraction treatments: a longterm comparison in patients with "borderline", equally susceptible Class II malocclusions. Am J Orthod Dentofacial Orthop 1994;105:444-449.
[9] Bonjardim LR, Gaviao MB, Carmagnani FG, Pereira LJ, Castelo PM. Signs and symptoms of temporomandibular joint dysfunction in children with primary dentition. J Clin Pediatr Dent 2003;28:53-58.

[10] Borstlap W, Stoelinga P, Hoppenreijs T, van't Hof M. Stabilisation of sagittal split advancement osteotomies with miniplates: a prospective, multicentre study with two-year follow-up. Part III - condylar remodelling and resorption. Int J Oral Maxillofac Surg 2004;33:649-55.

[11] Carlsson CR, Reid KI, Curran SL, Studts J, Ookeson JP, Falace D, et al. Psychological and physiological parameters of masticatory muscle pain. Pain 1998;76:297-307.

[12] Cascone P, Di Paolo C, Leonardi R, Pedulla E. Temporomandibular disorders and orthognathic surgery. J Craniofac Surg 2008;19:587-592.

[13] Chen CY, Palla S, Erni S, Sieber M, Gallo LM. Nonfunctional tooth contact in healthy controls and patients with myogenous facial pain. J Orofac Pain 2007;21:185-193.

[14] Chiappe G, Fantoni F, Landi N, Biondi K, Bosco M. Clinical value of 12 occlusal features for the prediction of disc displacement with reduction (RDC/TMD Axis I group IIa). J Oral Rehabil 2009;36:322-329.

[15] Corvo G, Tartaro G, Giudice A, Diomajuta A. Distribution of craniomandibular disorders, occlusal factors and oral parafunctions in a paediatric population. Eur J Paediatr Dent 2003;4:84-88

[16] Costen JB. A syndrome of ear and sinus symptoms dependent upon disturbed function of the temporomandibular joint. Ann Otol Rhinol Laryngol 1934;43:1-15.

[17] Dahl BL, Krogstad BS, Ogaard B, Eckersberg T. Signs and symptoms of craniomandibular disorders in two groups of 19-year-old individuals, one treated orthodontically and the other not. Acta Odontol Scand 1988;46:89-93.

[18] De Coster PJ, Van den Berghe LI, Martens LC. Generalized joint hypermobility and temporomandibular disorders: inherited connective tissue disease as a model with maximum expression. J Orofac Pain 2005; 19:47-57.

[19] Deguchi T, Uematsu S, Kawahara Y, Mimura H. Clinical evaluation of temporomandibular joint disorders (TMD) in patients treated with chin cup. Angle Orthod 1998;68:91-94.

[20] De Leeuw R, Bertoli E, Schmidt JE, Carlson CR. Prevalence of traumatic stressors in patients with temporomandibular disorders. J Oral Maxillofac Surg 2005;63:42-50.

[21] Deodato F, Trusendi R, Giorgetti R, Scalese MU. Predisposition for temporomandibular joint disorders: loose ligaments. Cranio 2006;24:179-183.

[22] Dibbets JM, Van der Weele LT. Extraction, orthodontic treatment, and craniomandibular dysfunction. Am J Orthod Dentofacial Orthop 1991;99:210-219.

[23] Dibbets JM, van der Weele LT. Long-term effects of orthodontic treatment, including extraction, on signs and symptoms attributed to CMD. Eur J Orthod $1992 ; 14: 16-20$

[24] Egermark I, Carlsson GE, Magnusson T. A prospective long-term study of signs and symptoms of temporomandibular disorders in patients who received orthodontic treatment in childhood. Angle Orthod 2005;75:645-650. 
[25] Ekberg EC, Nilner M. Treatment outcome of short- and long-term appliance therapy in patients with TMD of myogenous origin and tension-type headache. J Oral Rehabil 2006;33:713-721.

[26] Farella M, Michelotti A, Bocchino T, Cimino R, Laino A, Steenks MH. Effects of orthognathic surgery for class III malocclusion on signs and symptoms of temporomandibular disorders and on pressure pain thresholds of the jaw muscles. Int J Oral Maxillofac Surg 2007;36:583-587.

[27] Farella M, Michelotti A, Iodice G, Milani S, Martina R. Unilateral posterior crossbite is not associated with TMJ clicking in young adolescents. J Dent Res 2007;86:137-141.

[28] Forsell H, Kalso E. Application of principles of evidencebased medicine to occlusal treatment for temporomandibular disorders: are there lessons to be learned? J Orofac Pain 2004;18:9-22.

[29] Gameiro GH, Da Silva Aandrade A, Nouer DF, Ferraz de Arruda Veiga MC. How may stressful experience contribute to the development of temporomandibular disorders? Clin Oral Invest 2006;10:261-268.

[30] Gavish A, Halachmi M, Winocur E, Gazit E. Oral habits and their association with signs and symptoms of temporomandibular disorders in adolescent girls. J Oral Rehabil 2000;27:22-32.

[31] Gesch D, Bernardt O, Kirbschus A. Association of malocclusion and functional occlusion with temporomandibular disorders (TMD) in adults: a systematic review of population-based studies. Quintessence Int 2004;35:211221.

[32] Gianelly AA, Anderson CK, Boffa J. Longitudinal evaluation of condylar position in extraction and nonextraction treatment. Am J Orthod Dentofacial Orthop 1991;100:416-420.

[33] Glaros AG, Williams K, Lausten L, Friesen LR. Tooth contact in patients with temporomandibular disorders. Cranio 2005;23:188-193.

[34] Glaros AG, Williams K, Lausten L. The role of parafunctions, emotions and stress in predicting facial pain. J Am Dent Assoc 2005;136:451-458.

[35] Greene CS. The etiology of temporomandibular disorders: implications for treatment. J Orofac Pain 2001;15:93-105.

[36] Hagag G, Yoshida K, Miura H. Occlusion, prosthodontic treatment and temporomandibular disorders: a review. J Med Dent Sci 2000;47:61-66.

[37] Helenius LM, Hallikainen D, Helenius I, Meurman JH, Kononen M, Leirisalo-Repo $\mathrm{M}$, et al. Clinical and radiographic findings of the temporomandibular joint in patients with various rheumatic diseases. A case-control study. Oral Surg Oral Med Oral Pathol Oral Radiol Endod 2005;99:455-463.

[38] Henrikson T, Nilner M. Temporomandibular disorders, occlusion and orthodontic treatment. J Orthod 2003;30:129-137.

[39] Hirata RH, Heft MW, Hernandez B, King GJ. Longitudinal study of signs of temporomandibular disorders (TMD) in orthodontically treated and nontreated groups. Am J Orthod Dentofacial Orthop 1992;101:35-40.
[40] Hirsch C. No increased risk of temporomandibular disorders and bruxism in children and adolescents during orthodontic therapy. J Orofac Orthop 2009;70:39-50.

[41] How CK. Orthodontic treatment has little to do with temporomandibular disorders. Evid Based Dent 2004;5(3):75.

[42] Huddleston Slater JJ, Lobbezoo F, Onland-Moret NC, Naeije M. Anterior disc displacement with reduction and symptomatic hypermobility in the human temporomandibular joint: prevalence rates and risk factors in children and teenagers. J Orofac Pain 2007;21:55-62.

[43] Keeling SD, Garvan CW, King GJ, Wheeler TT, McGorray S. Temporomandibular disorders after early Class II treatment with bionators and headgears: results from a randomized controlled trial. Semin Orthod 1995;1:149-164.

[44] Klasser GD, Balasubramaniam R, Epstein J. Topical review - Connective tissue diseases: orofacial manifestations including pain. J Orofac Pain 2007;21:171-184.

[45] Klobas L, Tegelberg A, Axelsson S. Symptoms and signs of temporomandibular disorders in individuals with chronic whiplash-associated disorders. Swed Dent J 2004;28:29-36.

[46] Koh H, Robinson PG. Occlusal adjustement for treating and preventing temporomandibular joint disorders. J Oral Rehabil 2004;31:287-292.

[47] Köhler AA, Helkimo AN, Magnusson T, Hugoson A. Prevalence of symptoms and signs indicative of temporomandibular disorders in children and adolescents. A cross-sectional epidemiological investigation covering two decades. Eur Arch Paediatr Dent 2009;10(Suppl 1):16-25.

[48] Kremenak CR, Kinser DD, Harman HA, Menard CC, Jakobsen JR. Orthodontic risk factors for temporomandibular disorders (TMD). I: Premolar extractions. Am J Orthod Dentofacial Orthop 1992;101:13-20.

[49] Kremenak CR, Kinser DD, Melcher TJ, Wright GR, Harrison SD, Ziaja RR, et al. Orthodontics as a risk factor for temporomandibular disorders (TMD). II. Am J Orthod Dentofacial Orthop 1992;101:21-27.

[50] Larsson E, Rönnerman A. Mandibular dysfunction symptoms in orthodontically treated patients ten years after the completion of treatment. Eur J Orthod 1981;3:89-94.

[51] Luecke PE 3rd, Johnston LE Jr. The effect of maxillary first premolar extraction and incisor retraction on mandibular position: testing the central dogma of "functional orthodontics". Am J Orthod Dentofacial Orthop 1992;101:4-12.

[52] Luppanapornlarp S, Johnston LE Jr. The effects of premolar-extraction: a long-term comparison of outcomes in "clear-cut" extraction and nonextraction Class II patients. Angle Orthod 1993;63:257-272.

[53] Luther F. Orthodontics and the temporomandibular joint: where are we now? Part 1. Orthodontic treatment and temporomandibular disorders. Angle Orthod 1998;68:295-304.

[54] Macfarlane TV, Kenealy P, Kingdon HA, Mohlin BO, Pilley JR, Richmond S, et al. Twenty-year cohort study of health gain from orthodontic treatment: temporomandibular disorders. Am J Orthod Dentofacial Orthop 2009;135:692. el-e8. 
[55] Mac Namara JA, Seligman DA, Okeson P. Occlusion, orthodontic treatment and temporomandibular disorders: a review. J Orofac Pain 1995;9:73-90.

[56] Magnusson T, Egermarki I, Carlsson GE. A prospective investigation over two decades on signs and symptoms of temporomandibular disorders and associated variables. A final summary. Acta Odontol Scand 2005;63:99-109.

[57] Major P, Kamelchuk L, Nebbe B, Petrikowsky G, Glover K. Condyle displacement associated with premolar extraction and nonextraction orthodontic treatment of Class I malocclusion. Am J Orthod Dentofacial Orthop 1997;112:435-440.

[58] Matheus RA, Ramos-Perez FM, Menezes AV, Ambrosano GM, Halter-Neto F, Boscolo FN, et al. The relationship between temporomandibular dysfunction and head and cervical posture. J Appl Oral Sci 2009;17:204-208.

[59] McLaughlin RP, Bennett JC. The extraction-nonextraction dilemma as it relates to TMD. Angle Orthod 1995;65:175-186.

[60] Mohlin B, Axelsson S, Paulin G, Pietila T, Bondemark L, Brattström V, et al. TMD in relation to malocclusion and orthodontic treatment. Angle Orthod 2007;77:542-548.

[61] Mohlin BO, Derweduwen K, Pillery R, Kingdon A, Shaw WC, Kenealy P. Malocclusion and temporomandibular disorder: a comparison between adolescents with moderate to severe dysfunction with those without signs and symptoms of temporomandibular disorder and their further development to 30 years of age. Angle Orthod 2004;74:319-327.

[62] Munhoz WC, Marques AP. Body posture evaluations in subjects with internal temporomandibular joint derangement. Cranio 2009;27:231-242.

[63] Nilsson IM, List T, Drangsholt M. Incidence and temporal patterns of temporomandibular disorder pain among Swedish adolescents. J Orofac Pain 2007;21:127-132.

[64] Owen AH. Unexpected temporomandibular joint findings during fixed appliance therapy. Am J Orthod Dentofacial Orthop 1998;113:625-631.

[65] Pallegama RW, Ranasinghe AW, Weerasinhe VP, Sitheeque MAM. Anxiety and personality traits in patients with muscle related temporomandibular disorders. J Oral Rehabil 2005;32:701-705

[66] Paquette DE, Beattie JR, Johnston LE Jr. A long-term comparison of nonextraction and premolar extraction edgewise therapy in "borderline" Class II patients. Am J Orthod Dentofacial Orthop 1992;102:1-14.

[67] Pellizoni SE, Salioni MA, Juliano Y, Guimares AS, Alonso LG. Temporomandibular joint disc position and configuration in children with functional unilateral posterior crossbite: a magnetic resonance imaging evaluation. Am J Orthod Dentofacial Orthop 2006;129:785-793.

[68] Popowich K, Nebbe B, Major PW. Effect of Herbst treatment on temporomandibular joint morphology: a systematic literature review. Am J Orthod Dentofacial Orthop 2003;123:388-94.

[69] Pullinger A, Hollender L. Assessment of mandibular condyle position: a comparison of transcranial radiographs and linear tomograms. Oral Surg Oral Med Oral Pathol 1985;60:329-334.
[70] Pullinger AG, Seligman DA, Gornbein JA. A multiple logistic regression analysis of the risk and relative odds of temporomandibular disorders as a function of common occlusal features.J Dent Res 1993;72:968-979.

[71] Rendell JK, Norton LA, Gay T. Orthodontic treatment and temporomandibular joint disorders. Am J Orthod Dentofacial Orthop 1992;101:84-87.

[72] Rey D, Oberti G, Baccetti T. Evaluation of temporomandibular disorders in Class III patients treated with mandibular cervical headgear and fixed appliances. Am J Orthod Dentofacial Orthop 2008;133:379-381.

[73] Rheinhardt R, Tremel T, Wehrbein H, Reinhardt W. The unilateral chewing phenomenon, occlusion, and TMD. Cranio 2006;24:166-170.

[74] Robin O, Carpentier P. Données actuelles sur le traitement des algies et dysfonctionnement de l'appareil manducateur. Le chirurgien dentiste de France 2006;12631264:29-41.

[75] Rossetti LM, Rossetti PH, Conti PC, de Araujo Cdos R. Association between sleep bruxism and temporomandibular disorders: a polysomnographic pilot study. Cranio 2008;26:16-24

[76] Ruf S, Pancherz H. Long-term TMJ effects of Herbst treatment: a clinical and MRI study. Am J Orthod Dentofacial Orthop 1998;114:475-483.

[77] Ruf S, Pancherz H. Temporomandibular joint remodeling in adolescents and young adults during Herbst treatment: A prospective longitudinal magnetic resonance imaging and cephalometric radiographic investigation. Am J Orthod Dentofacial Orthop 1999;115:607-618.

[78] Sadowsky C, Begole EA. Long-term status of temporomandibular joint function and functional occlusion after orthodontic treatment. Am J Orthod 1980;78:201-212.

[79] Sadowsky C, Polson AM. Temporomandibular disorders and functional occlusion after orthodontic treatment: results of two long-term studies. Am J Orthod 1984, 86:386-390.

[80] Sadowsky C, Theisen TA, Sakols EI. Orthodontic treatment and temporomandibular joint sounds - a longitudinal study. Am J Orthod Dentofacial Orthop 1991;99:441-447.

[81] Sato F, Kino K, Sugisaki M, Haketa T, Amenori Y, Ishikawa $\mathrm{T}$, et al. Teeth contacting habit as a contributing factor to chronic pain in patients with temporomandibular disorders. J Med Dent Sci 2006;53:103-109.

[82] Toscano P, Defabianis P. Clinical evaluation of temporomandibular disorders in children and adolescents: a review of the literature. Eur J Paediatr Dent 2009;10:188-192

[83] Tsukiyama Y, Baba K, Clark GT. An evidence-based assessment of occlusal adjustment as a treatment of temporomandibular disorders. J Prosthet Dent 2001;86:57-66.

[84] Turner J, Deyo R, Loeser J, Von Korff M, Fordyce WE. The importance of placebo effects in pain treatment and research. JAMA 1994;271:1609-1614.

[85] Van Selms MK, Lobbezoo F, Visscher CM, Naeije M. Myofascial temporomandibular disorder pain, parafunctions and psychological stress. J Oral Rehabil 2008;35:45-52. 
[86] Visscher C, Hofman N, Mes C, Lousberg R, Naeije M. Is temporomandibular pain in chronic whiplash-associated disorders part of a more widespread pain syndrome? Clin J Pain 2005;21:353-357.

[87] Wahlund K. Temporomandibular disorders in adolescents. Epidemiological and methodological studies and a randomized controlled trial. Swed Dent J Suppl. 2003;164:2-64.

[88] White C, Dolwick M. Prevalence and variance of temporomandibular dysfunction in orthognathic surgery patients. Int J Adult Orthod Orthognath Surg 1992;7:7-14.

[89] Williamson EH. Temporomandibular dysfunction and repositioning splint therapy. Prog Orthod 2005;6:206-213.

[90] Witzig JW, Spahl TJ. The clinical management of basic maxillofacial orthopedic appliances. Volume 1: Mechanics. Boston: PSG Publishing 1987:156.
[91] Wolford LM, Reiche-Fischel O, Mehra P. Changes in temporomandibular joint dysfunction after orthognathic surgery. J Oral Maxillofac Surg 2003;61:655-660; discussion 661

[92] Wyatt WE. Preventing adverse effects on the temporomandibular joint through orthodontic treatment. Am J Orthod Dentofacial Orthop 1987;91:493-499.

[93] Yap AUJ, Tan KBC, Chua EK, Tan HH. Depression and somatization in patients with temporomandibular disorders. J Prosthet Dent 2002;88:479-484.

[94] Yun P, Kim Y. The role of facial trauma as a possible etiologic factor in temporomandibular joint disorder. J Oral Maxillofac Surg 2005;63:1576-1583.

[95] Zimmer B, Schwestka R, Kubein-Meesenburg D. Changes in mandibular mobility after different procedures of orthognathic surgery. Eur J Orthod 1992;14:188-197. 